\title{
MANEJO DE PACIENTES COM HALITOSE ATENDIDOS NO SERVIÇO PÚBLICO DE SAÚDE
}

\section{MANAGEMENT OF PATIENTS WITH HALITOSIS ON PUBLIC HEALTH SERVICE}

\begin{abstract}
Luciana Mara Peixôto Araujo*, Ariane de Oliveira Santana**, lasmin de Sousa Moura**, Jefferson David Melo De-Matos**, Marignês Theotonio dos Santos Dutra***, Carolina Carvalho de Oliveira Santos****, Thiago Fonseca-Silva******
\end{abstract}

Autor para correspondência: Thiago Fonseca-Silva - thiagofonsecasilva@gmail.com

*Professora de Periodontia; Centro Universitário Leão Sampaio - UNILEÃO; Juazeiro do Norte, Ceará.

**Alunos de graduação em Odontologia; Centro Universitário Leão Sampaio - UNILEÃO; Juazeiro do Norte, Ceará.

***Professora do Curso de Capacitação ao Atendimento ao Portador de Halitose - Associação Brasileira de Halitose - ABHA.

****Professora do Departamento de Odontologia Restauradora; Universidade Federal do Paraná - UFPR; Curitiba, Paraná.

*****Professor da Área de Diagnóstico Bucal; Centro Universitário Leão Sampaio - UNILEÃO; Juazeiro do Norte, Ceará.

\section{R E S U M O}

\begin{abstract}
A halitose é caracterizada pelo odor fétido emanado pela cavidade bucal considerado um sintoma constrangedor com significativo impacto social. O objetivo do presente estudo foi avaliar o conhecimento dos profissionais atuantes no Sistema Único de Saúde - SUS (Atenção Primária e Secundária) sobre a halitose, bem como analisar os critérios para encaminhamentos dos casos suspeitos. $O$ presente estudo contou com a participação de 37 profissionais de saúde entre médicos e cirurgiões-dentistas. Todos os participantes responderam um questionário estruturado sobre a etiologia, manejo e tratamento da halitose. Entre todos os profissionais analisados $92 \%(n=34)$ afirmaram receber pacientes com queixa de halitose. Para $88 \%(n=22)$ dos cirurgiões-dentistas e $50 \%(n=06)$ dos médicos a principal causa da halitose advém de origem bucal. Do total de participantes, 89,1\% $(n=33)$ afirmaram realizar o tratamento da halitose. Em adição, o exame complementar mais solicitado para o diagnóstico da halitose foi a radiografia da face. Os resultados sugerem que existem incoerências no manejo e tratamento da halitose no setor público de saúde. O presente estudo traz à tona a necessidade de conscientizar e qualificar os médicos e cirurgiões-dentistas quanto aos fatores etiológicos, formas de diagnóstico e tratamento da halitose.
\end{abstract}

PALAVRAS-CHAVE: Halitose. Diagnóstico. Saúde Pública. 
Halitosis is characterized by a foul odor emanating from the oral cavity. This condition is considered an embarrassing symptom with a significant social impact for the individual. The aim of this study was to evaluate the knowledge of professionals working on public health service about halitosis and to examine the criteria for referrals of suspected cases. This study had the participation of 37 health professionals including medical doctors and dentists. All participants answered a questionnaire about the etiology, management and treatment of halitosis. Among all the professionals analyzed $92 \%(n=34)$ reported receiving patients complaining of halitosis. $88 \%(n=22)$ of dentists and $50 \%(n=06)$ of medical doctors believe that the main cause of halitosis has an oral origin. $89.1 \%(n=33)$ of samples reported performing the treatment of halitosis. In addition, the complementary exam most requested for the diagnosis of halitosis was the radiograph of the face. The results suggest that there is a mistake in the management of patients with halitosis attended in the public health service. This study brings to light the need to educate and train medical doctors and dentists about the etiological factors, forms of diagnosis and treatment of halitosis.

KEYWORDS: Halitosis. Diagnosis. Public Health. 


\section{INTRODUÇÃO}

A halitose, também designada hálito fétido ou mau hálito, é uma condição caracterizada pela emanação de odores fétidos pela boca com prevalência estimada entre 10 a $30 \% \%^{1,2}$. A literatura descreve que $87 \%$ dos fatores etiológicos da halitose são originados na cavidade bucal ou em áreas relacionadas como o ouvido, nariz e garganta e $13 \%$ são de origem extra bucal como desordens do trato respiratório ou gastrointestinal ${ }^{3}$.

Má higiene bucal, gengivite e periodontite, saburra lingual, afecções no nariz e garganta (amigdalite, sinusite, presença de corpos estranhos e rinite), diminuição da saliva, tabagismo e etilismo são os principais fatores associados à halitose ${ }^{3}$. As desordens do trato gastrointestinal são consideradas condições raramente associadas ${ }^{4}$. Além disso, algumas desordens sistêmicas também são apontadas como fatores etiológicos associados à halitose. Nesse sentido a diabetes é descrita na literatura como uma das condições sistêmicas associadas ao mau hálito ${ }^{5}$. Os portadores dessa condição podem apresentar acúmulo de corpos cetônicos na corrente sanguínea (cetoacidose), os quais são expirados e liberados na saliva favorecendo a formação do hálito cetónico (hálito com odor semelhante ao de frutas podres) ${ }^{5-7}$.

A literatura descreve que indivíduos com queixa de halitose possuem tendência de diminuição da autoconfiança e apresentam insegurança nas relações interpessoais e sociais ${ }^{8,9}$. $O$ correto diagnóstico e tratamento desta condição podem melhorar a qualidade de vida desses indivíduos', entretanto, incapacidade de auto perceber o problema pode acarretar no não diagnóstico da halitose ${ }^{8,10}$. Nesse sentido, a identificação e encaminhamento de indivíduos com halitose por profissionais de saúde, passa a configurar uma importante ferramenta auxiliar ao diagnóstico e tratamento desta condição.

O objetivo do presente estudo foi avaliar o conhecimento dos profissionais atuantes no Sistema Único de Saúde - SUS (Atenção Primária e Secundária) sobre a halitose, bem como, analisar os critérios para encaminhamento dos casos suspeitos.

\section{MATERIAIS E MÉTODOS}

O presente estudo, do tipo transversal descritivo, contou com uma amostra de conveniência de 37 profissionais de saúde entre médicos e cirurgiõesdentistas atuantes no Sistema Único de Saúde - SUS (Atenção Primária e Secundária) de um município da região sul do estado do Ceará. Para a execução do presente trabalho, o mesmo contou com a aprovação do Comitê de Ética em Pesquisa, protocolo $\mathrm{n}^{\circ}$ 1.021 .963 (CAAE: 43623715.8 .0000 .5048 ).

A população alvo do estudo foi composta de todos os profissionais médicos e cirurgiões-dentistas atuantes no SUS no município sede da pesquisa. O serviço público de saúde deste município é composto atualmente por 20 equipes de Estratégia de Saúde da Família (ESF), 01 Centro de Especialidades Odontológicas Municipal, 01 Centro de Especialidades Odontológicas Regional e uma Policlínica Médica. Um total de 55 profissionais (22 médicos e 33 cirurgiões-dentistas) foram convidados a participar do trabalho. Somente os profissionais que aceitaram participar do estudo e assinaram o termo de consentimento livre esclarecido (TCLE) foram incluídos no trabalho. A seleção da amostra contou com a inclusão de 25 cirurgiões-dentistas, sendo 13 profissionais da atenção primária e $12 \mathrm{da}$ atenção secundária. Adicionalmente foram incluídos 12 profissionais médicos, sendo 10 médicos atuantes na atenção primária e 02 na atenção secundária (01 Otorrinolaringologista e 01 Gastroenterologista). A contribuição dos participantes deu-se através do preenchimento de um questionário estruturado sobre a etiologia e tratamento da halitose, bem como, dos encaminhamentos dos indivíduos (Tabela 01 ). 


\section{PERGUNTAS}

01. Qual é sua profissão?

02. Você recebe pacientes com queixa de halitose ou alguma sensação de mau gosto ou cheiro na boca?

03. Para você, qual a principal causa da halitose?

04. Você trata halitose?

05. Se você trata halitose, qual é o tipo de tratamento?

06. Você solicita algum tipo de exame para auxiliar o diagnóstico da halitose?

07. Você costuma encaminhar os pacientes com suspeita de halitose?

08. Se você encaminha o paciente com suspeita de halitose, o referenciamento é para qual profissional da saúde?

Todos os dados obtidos foram digitados e tabulados no programa SPSS (StatisticalPackage for Social Science) versão 17.0 e posteriormente tratados com testes estatísticos descritivos.

\section{RESULTADOS}

Dos 37 participantes da pesquisa $68 \%(n=25)$ eram cirurgiões-dentistas, dos quais, 13 atuavam na Atenção Primária à Saúde e 12 na Secundária. Os médicos participantes representaram 32\% $(n=12)$ da amostra, dos quais, 10 atuavam na Atenção Primária à Saúde e 02 na Secundária (Otorrinolaringologista e Gastroenterologista).

De todos os profissionais analisados, $92 \%(n=34)$ afirmaram receber pacientes com queixa de halitose. Do total de cirurgiões-dentistas incluídos no estudo $88 \%(n=22)$ afirmaram que a principal causa da halitose dos pacientes está relacionada à cavidade bucal. Para os médicos, a cavidade bucal e os distúrbios gástricos $150 \%$ e $41,66 \%$ respectivamente) são as principais causas da halitose (tabela 02).

Tabela 2. Principais causas da halitose classificada pelos profissionais de saúde.

\begin{tabular}{lcr}
\hline PRINCIPAIS CAUSAS & CIRURGIÃO DENTISTA & \multicolumn{1}{c}{ MÉDICO } \\
\hline Origem bucal & $88 \%(n=22)$ & $50 \%(n=6)$ \\
Desordens Gástrica & $12 \%(n=03)$ & $41,66 \%(n=5)$ \\
Desordens respiratórias & $0,0 \%(n=0)$ & $8,33 \%(n=1)$ \\
\hline
\end{tabular}

Dos 37 profissionais incluídos no presente estudo, $89,1 \%(n=33)$ afirmaram realizar o tratamento da halitose; sendo, $92 \%(n=23)$ dos cirurgiões-dentistas e $83,33 \%(n=10)$ dos médicos. Os cirurgiõesdentistas relataram realizar o tratamento da halitose por meio da adequação do meio bucal (tratamento periodontal básico, remoção de restos radiculares, selamento cavitário, instrução de higiene bucal e da prótese); os médicos relataram realizar o tratamento da halitose com a utilização de enxaguantes bucais, antifúngicos, antiácidos, antibióticos e instrução de higiene bucal.

Em relação aos exames complementares solicitados pelos profissionais que relataram tratar halitose, $32,0 \%(n=08)$ dos cirurgiões-dentistas afirmaram solicitar radiografias da face (radiografias 
panorâmicas ou dos seios da face) e $42,0 \%(n=5)$ dos médicos relataram solicitar o exame de endoscopia para auxiliar no diagnóstico da halitose (Figura 01).

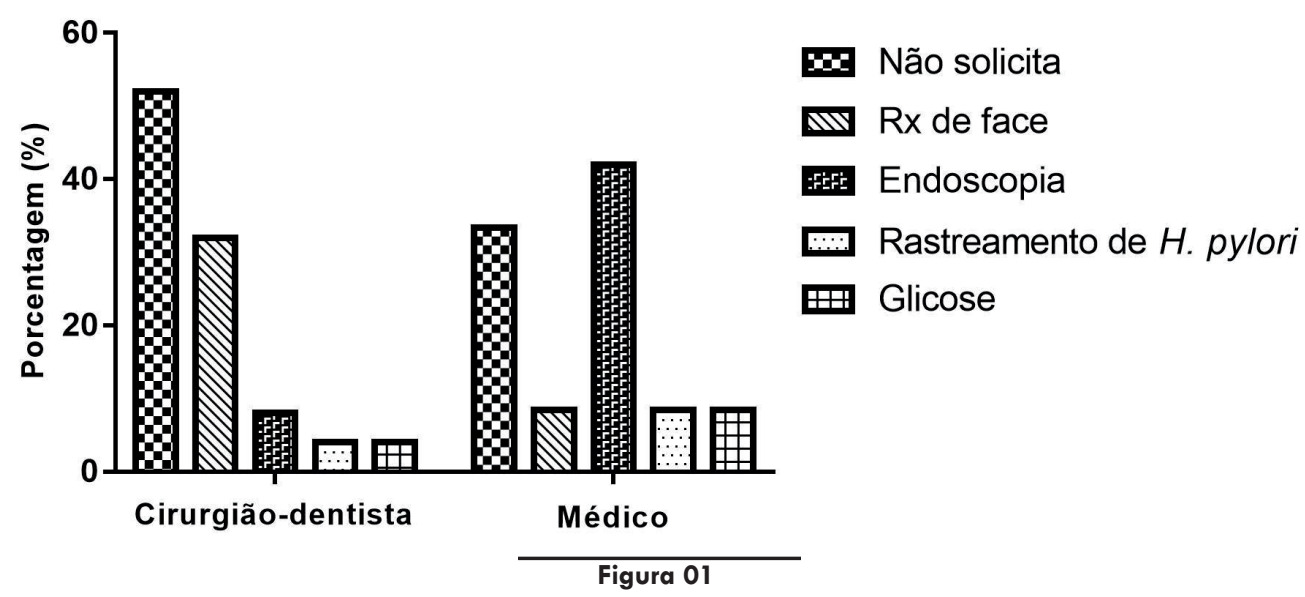

Quanto ao encaminhamento, $75,6 \%(n=28)$ dos participantes da pesquisa afirmaram encaminhar os pacientes com halitose para outros profissionais de saúde sendo $76,0 \%(n=19)$ dos cirurgiões-dentistas e $75,0 \%$ $(n=09)$ dos médicos. A maioria dos cirurgiões-dentistas 36,0\%, $(n=09)$ encaminham os pacientes para 0 gastroenterologista; enquanto $50,0 \%(n=06)$ dos médicos encaminham esses pacientes aos cirurgiões-dentistas (Figura 02).

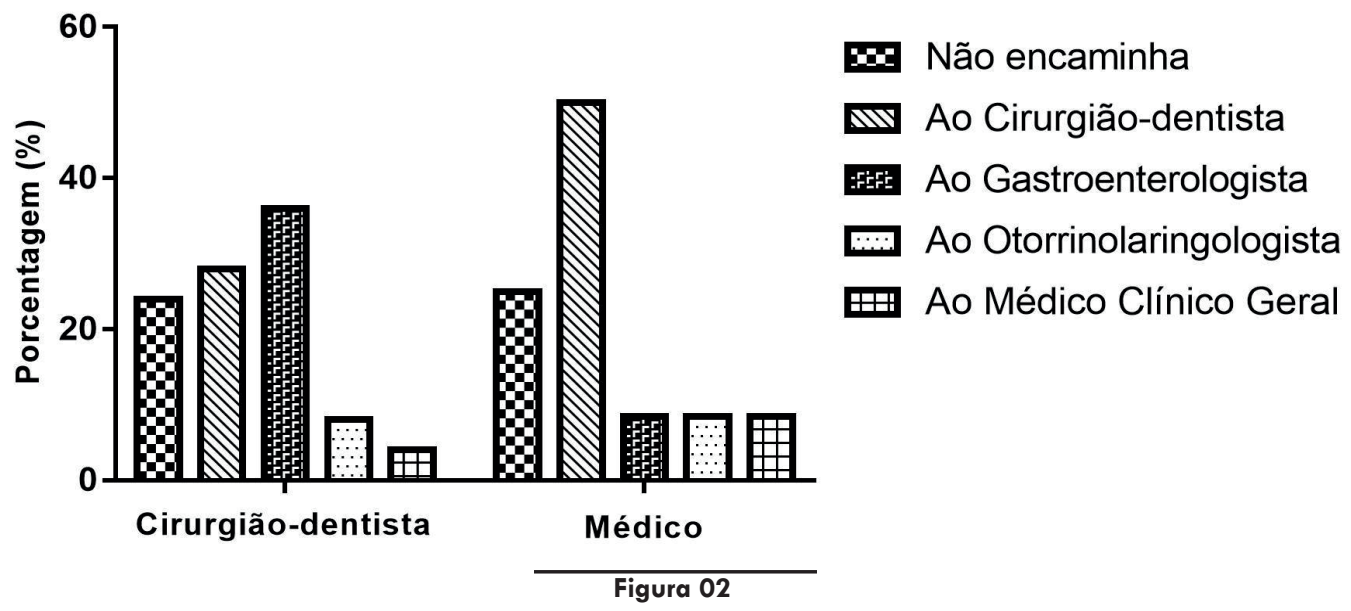

\section{DISCUSSÃO}

A halitose desperta $\circ$ interesse de muitos profissionais da área de saúde em virtude da ocorrência bastante significativa na população em geral. A literatura descreve que a halitose possui prevalência variável entre 10 e $30 \%$ de acometimento populacional ${ }^{3,8,10-12}$.

Diversos autores discutem que halitose não seria uma condição patológica específica, mas sim, um sinal indicativo de outras manifestações patológicas/ fisiológicas ou de precária higiene bucal ${ }^{13}$. Dentre as causas da halitose a má higiene bucal, saburra lingual, amigdalites e sinusites parecem ser as causas mais comuns do problema ${ }^{3}$. A literatura ressalta que $87 \%$ dos casos de halitose possuem seu fator etiológico fundamentado na cavidade bucal ${ }^{3,11,14}$. Os fatores intra bucais parecem estar relacionados à formação do biofilme dental cuja microbiota é principalmente anaeróbica gran-negativa, com grande potencial para a formação de compostos de enxofre, que são os componentes mais comumente associados aos gases representativos da halitose ${ }^{15}$. 
A literatura descreve que indivíduos portadores de halitose possuem as relações interpessoais dificultadas devido ao odor fétido do hálito ${ }^{1,39,16}$. Tal situação é responsável por prejuízos na vida social e profissional do sujeito". O indivíduo portador de halitose, no geral, possui uma incapacidade de auto perceber $\circ$ problema e consequentemente trata$10^{16}$. Nesse sentido os cirurgiões-dentistas, médicos e enfermeiros possuem um papel fundamental na identificação de pacientes com halitose. Entretanto, a falta de informações a cerca dos fatores etiológicos, formas de diagnósticos e tipos de tratamento para a halitose torna-se um complicador para que os profissionais de saúde identifiquem a condição.

Nossos resultados sugerem que, de maneira geral, os participantes da pesquisa reconhecem a cavidade bucal como sendo o principal sítio etiológico para a halitose. A boca é responsável por $87 \%$ dos fatores etiológicos da halitose ${ }^{3,11}$, desta forma, o cirurgiãodentista é o profissional de saúde mais preparado para estabelecer 0 diagnóstico e tratamento da halitose ${ }^{15,17-19}$. A análise dos encaminhamentos realizados pelos profissionais estudados, demostrou que $50 \%$ dos médicos encaminham os pacientes suspeitos para os dentistas. Tal fato, estabelece uma coerência inter profissional para a definição diagnóstica, bem como, para o tratamento desta condição. Entretanto, a maioria dos cirurgiõesdentistas encaminham os pacientes suspeitos de apresentarem halitose para 0 especialista em gastrenterologia. Tal fato demonstra um contrassenso entre os cirurgiões-dentistas e os encaminhamentos realizados por esses profissionais, o que pode ser resultante da falta de embasamento científico acerca da halitose. Carvalho, et al ${ }^{15}$, (2008) ressaltaram que os cursos de formação profissional em Odontologia, nível graduação, abordam o tema halitose de forma superficial. Tal fato poderia impactar negativamente nas habilidades e competências dos profissionais quando do diagnóstico e tratamento dos casos de halitose.

$\mathrm{Na}$ busca de solucionar a queixa dos pacientes, observou-se, que médicos e dentistas focam suas hipóteses de diagnóstico em uma única possibilidade, - que os levam a solicitar exames complementares de auto custo aos serviços de saúde como endoscopias e radiografias de face. Tendo em vista os fatores etiológicos da halitose, as causas bucais deveriam ser as primeiras investigadas ${ }^{11,13,17,19,20 .}$ Uma boa anamnese e exame físico intra bucal são ferramentas importantes na identificação de fatores bucais associados à halitose ${ }^{13,15,17,19}$. Uma avaliação clínica minuciosa seria capaz de detectar a presença de placa bacteriana, restos radiculares, dentes cavitados, saburra lingual, doença periodontal, que são importantes fatores associados à formação de compostos odoríferos e consequentemente relacionados à halitose ${ }^{14,21,22}$.

O tratamento da halitose deve ser baseado no combate a causa que determina a produção dos gases voláteis causadores do mau odor ${ }^{15}$. Nossos resultados evidenciaram que os cirurgiões-dentistas que se dispunham a fazer o tratamento da halitose - faziam por meio da remoção dos possíveis fatores etiológicos associados à condição como o tratamento periodontal básico, remoção de restos radiculares, selamento cavitário, instrução de higiene bucal e de próteses. É importante destacar que escovação dentária correta, limpeza da língua e uso de fio dental são estratégias relevantes no combate à halitose ${ }^{15,19,22}$. Os médicos, quando se dispunham a tratar a halitose, o faziam, talvez, de forma empírica com a prescrição de enxaguantes bucais, antifúngicos, antiácidos e antibióticos. Vale ressaltar que nenhuma dessas formas de tratamento atuariam de forma efetiva nos fatores etiológicos da halitose ${ }^{14,23}$.

\section{CONSIDERAÇÕES FINAIS}

A partir dos dados do presente estudo podese sugerir que existem incoerências no manejo e tratamento da halitose no setor público de saúde na amostra estudada. $O$ presente estudo traz à tona a necessidade de conscientizar e qualificar médicos e cirurgiões-dentistas quanto aos fatores etiológicos, formas de diagnóstico e tratamento da halitose.

\section{REFERÊNCIAS}

1. de Jongh $A$, van Wijk AJ, Horstman $M$, de Baat $C$. Self-perceived halitosis influences social interactions. BMC Oral Health. 2016;16(1):31. doi: 10.1186/s1 2903-016-0189-9. 
2. Scully C, Greenman J. Halitosis (breath odor). Periodontol 2000. 2008;48:66-75. doi: $10.1111 /$ i.1600-0757.2008.00266.x

3. Madhushankari GS, Yamunadevi A, Selvamani M, Mohan Kumar KP, Basandi PS. Halitosis - An overview: Part-I - Classification, etiology, and pathophysiology of halitosis. J Pharm Bioallied Sci. 2015;7(Suppl 2):S339-S343. doi: 10.4103/09757406.163441

4. Fedorowicz Z, Aljufairi $H$, Nasser M, Outhouse TL, Pedrazzi V. Mouthrinses for the treatment of halitosis. Cochrane Database Syst Rev. 2008;(4):CD006701. doi: 10.1002/14651858. CD006701.pub2

5. Negrato CA, Tarzia O. Buccal alterations in diabetes mellitus. Diabetol Metab Syndr. 2010;2:3. doi: $10.1186 / 1758-5996-2-3$

6. Dal Rio AC, Nicola EM, Teixeira AR. Halitosis-an assessment protocol proposal. Braz J Otorhinolaryngol. 2007;73(6):835-842.

7. Mendes D, Carneiro M, Filho J, Caetano MR, Leite $M$, Rodrigues $C$. Doença Periodontal e diabetes na percepção de adultos: um estudo qualitativo. Rev Uni Cient. 2013;15(1):51-61.

8. Hammad MM, Darwazeh AM, Al-Waeli H, Tarakii B, Alhadithy TT. Prevalence and awareness of halitosis in a sample of Jordanian population. J Int Soc Prev Community Dent. 2014;4(Suppl 3):S178-186. doi: 10.4103/2231-0762.149033

9. Suzuki N, Yoneda M, Naito T, Iwamoto T, Hirofuji T. Relationship between halitosis and psychologic status. Oral Surg Oral Med Oral Pathol Oral Radiol Endod. 2008; 106(4):542-547. doi: 10.1016/i.tripleo.2008.03.009

10. Spouge JD. Halitosis, A review of its causes and treatment. Dent Pract Dent Rec. 1964;1 4:307-317.

11. Rosing CK, Loesche W. Halitosis: an overview of epidemiology, etiology and clinical management. Braz Oral Res. 2011 ;25(5):466-471.

12. Nadanovsky P, Carvalho LB, Ponce de Leon A. Oral malodour and its association with age and sex in a general population in Brazil. Oral Dis. 2007;13(1):105-109. doi: 10.1111/i.16010825.2006.01257.x

13. Yaegaki K, Coil JM. Examination, classification, and treatment of halitosis; clinical perspectives. J Can Dent Assoc. 2000;66(5):257-261.

14. Putt MS, Yu D, Kohut BE. Inhibition of calculus formation by dentifrice formulations containing essential oils and zinc. Am J Dent. 2002;15(5):335338.

15. Carvalho MF, Rodrigues PA, Chaves MGAM. Halitose: revisão literária. HU Revista. 2008;34(4):273-279.

16. Veeresha KL, Bansal M, Bansal V. Halitosis: A frequently ignored social condition. J Int Soc Prev Community Dent. 2011 ; 1 (1):9-13. doi: $10.4103 / 2231-0762.86374$

17. Amorim JA, Lins RDAU, Souza AD, Alves RD, Maciel MAS, N. LR. Aspectos epidemiológicos e etiológicos da halitose: considerações recentes. Rev. bras. odontol. 2010;67(1):76-80.

18. Rio ACCD, Nicola EMD, Teixeira ARF. Halitose: proposta de um protocolo de avaliação. Rev. Bras. Otorrinolaringol. 2007;73(6):835-842.

19. Uliana RMB, Briques W. Halitose conceitos básicos sobre, diagnóstico, microbiologia, causas, tratamento. . $15^{\circ}$ Conclave Odontológico Internacional de Campinas. 2003;1:1-8.

20. Aylikci BU, Colak H. Halitosis: From diagnosis to management. J Nat Sci Biol Med. 2013;4(1):14-23. doi: 10.4103/0976-9668.107255

21. Rosing CK, Gomes SC, Bassani DG, Oppermann RV. Effect of chewing gums on the production of volatile sulfur compounds (VSC) in vivo. Acta Odontol Latinoam. 2009;22(1):11-14.

22. Kara C, Tezel A, Orbak R. Effect of oral hygiene instruction and scaling on oral malodour in a population of Turkish children with gingival inflammation. Int J Paediatr Dent. 2006;16(6):399404.

23. van den Broek $A M$, Feenstra $L$, de Baat $C$. 
A review of the current literature on aetiology

and measurement methods of halitosis. J

Dent. 2007;35(8):627-635. doi: 10.1016/i.

jdent.2007.04.009 